\title{
Fragile topologically protected perfect reflection for acoustic waves
}

\author{
Chang-Yin Ji, ${ }_{1}^{1}$ Yongyou Zhang $\odot,{ }^{1, *}$ Yunhong Liao, ${ }^{2}$ Xiaoming Zhou, ${ }^{2}$ Jian-Hua Jiang, ${ }^{3}$ Bingsuo Zou, ${ }^{1}$ and Yugui Yao ${ }^{1,+}$ \\ ${ }^{1}$ Key Lab of Advanced Optoelectronic Quantum Architecture and Measurement (MOE), \\ Beijing Key Lab of Nanophotonics \& Ultrafine Optoelectronic Systems, \\ and School of Physics, Beijing Institute of Technology, Beijing 100081, China \\ ${ }^{2}$ Key Laboratory of Dynamics and Control of Flight Vehicle, Ministry of Education and School of Aerospace Engineering, \\ Beijing Institute of Technology, Beijing 100081, People's Republic of China \\ ${ }^{3}$ School of Physical Science and Technology, and Collaborative Innovation Center of Suzhou Nano Science and Technology, \\ Soochow University, Suzhou, China
}

(Received 4 October 2019; accepted 13 December 2019; published 6 February 2020)

\begin{abstract}
Fragile topology is firstly demonstrated in acoustic crystals and then a realistic scheme is proposed to manipulate the transport of acoustic topological edge states (ATESs), i.e., by coupling them with side acoustic cavities. We find that single-mode cavities can completely flip the ATES pseudospin to form a perfect reflection, as long as their resonant frequencies fall into the topological band gap. The perfect reflection of the ATESs is protected by the fragile topology, which is proved by the one-dimensional topological waveguide-cavity transport theory. This fragile topologically protected perfect reflection is immune to the conventional defects (such as bending and disorder) and provides a realistic paradigm for manipulating the ATES transport. As examples, two potential applications, i.e., distance sensors and acoustic switches, are proposed based on the perfect reflection.
\end{abstract}

DOI: 10.1103/PhysRevResearch.2.013131

\section{INTRODUCTION}

Controlling and tuning the transport of acoustic waves are crucially important for fundamental acoustics and a broad range of applications [1-21]. Acoustic crystals are a class of artificially engineered metamaterials with fascinating properties, which provide a powerful and flexible scheme to confine, guide, and reflect acoustic waves, thereby forming cavities, waveguides, and mirrors [22-29]. Coupled architectures of acoustic cavities and waveguides are favorable for many prominent acoustic devices, such as switches, filters, and logic elements [30-34]. However, the quality of these acoustic devices is very sensitive to the fabrication defects, that is, the state-of-the-art acoustic devices show strong dependence on manufacturing capability. Introducing topological concepts into acoustics not only opens a new chapter in manipulating the transport of acoustic waves, but also provides a novel research perspective to overcome the vexing degradation of device performance due to unavoidable defects [35-69]. Nontrivial bulk bands make the transport of topological edge states be robust to defects and disorders in devices. Some topological prototype devices have been proposed with brand new properties, such as high transmission waveguides [35-37,39,42,50], vortex chirality [43], beam separation [49], delay lines [59], directional acoustic antennas [68], lasers

\footnotetext{
*Corresponding author: yyzhang@bit.edu.cn

†ygyao@bit.edu.cn

Published by the American Physical Society under the terms of the Creative Commons Attribution 4.0 International license. Further distribution of this work must maintain attribution to the author(s) and the published article's title, journal citation, and DOI.
}

[70-72], and so on. They are based on the topologically protected perfect transmission.

From an inverse view, one may wonder whether there exists a topologically protected perfect reflection (TPPR) being robust against impurities. For this unprecedented realm, we find the answer is true once we turn to the fragile topological acoustic crystals. The fragile topology is a new concept recently introduced into electronic and classical-wave systems [73-79], which can be trivialized by adding atomic insulators. However, it is still elusive for the strong influence on the transport of the topological edge states (TESs) due to the fragile topology. In the present work, we reveal that singlemode cavities can perfectly flip the pseudospin of the acoustic TESs (ATESs) in fragile topological acoustic insulators as long as their resonant frequencies fall into the topological band gap. Accordingly, single-mode cavities can lead to a complete back reflection for the ATES transport, i.e., the prefect reflection. One step further, numerical results demonstrate that the perfect reflection is robust against conventional fabrication defects, such as disorders and bending interfaces, which is the character of the TPPR and does not exist in the trivial waveguides coupled to cavities. Conceptually, the TPPR provides a realistic scheme to control and tune the transport of the ATESs and thus it is favorable for designing acoustic topological devices. As an example, the potential application to sensors and switches are discussed, which may bring about remarkable values for the field of topological acoustic devices.

The following work is organized as follows. In Sec. II, a fragile acoustic topological insulator is designed to show the TPPR. In Sec. III, a quantum model is built to describe the TPPR and related application to sensor and switch is discussed in Sec. IV. Finally, a brief conclusion is given in Sec. V. 

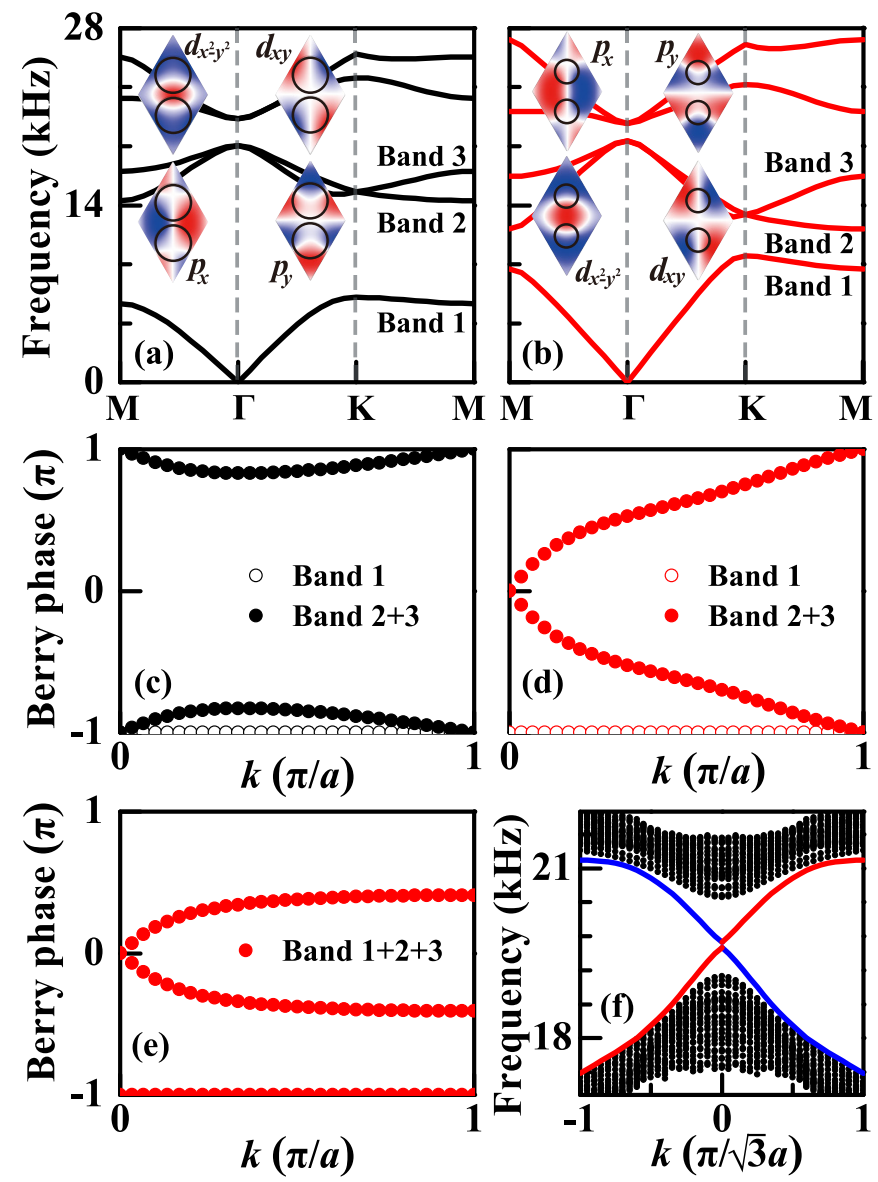

FIG. 1. Band structures for (a) trivial and (b) topological graphenelike acoustic crystals with $r=0.45 \mathrm{~cm}$ and $0.3 \mathrm{~cm}$, respectively. Insets in (a) and (b) are the acoustic wave modes of $p_{x}, p_{y}, d_{x y}$, and $d_{x^{2}-y^{2}}$ orbitals at $\Gamma$ point. (c) and (d) Evolution of Berry phase for the bottom three bands in (a) and (b), calculated separately. (e) Evolution of Berry phase for the bottom three bands in (b), calculated together. (f) Topological edge bands for armchair boundary between the trivial and topological acoustic insulators whose bulk bands are in (a) and (b), respectively.

\section{TOPOLOGICALLY PROTECTED PERFECT REFLECTION}

The considered fragile acoustic topological insulator (ATI) is graphene-like with lattice constant $a=\sqrt{3} \mathrm{~cm}$ and circular solid rod radius $r$. Numerical calculations are implemented by finite element method through this work. The solid rods are the stainless steel with the longitudinal sound speed of $6.010 \mathrm{~km} / \mathrm{s}$ and mass density of $7.800 \times 10^{3} \mathrm{~kg} / \mathrm{m}^{3}$. The steel rods are embedded in the background air whose sound speed and mass density are taken as $0.343 \mathrm{~km} / \mathrm{s}$ and $1.250 \mathrm{~kg} / \mathrm{m}^{3}$, respectively. For such acoustic crystals, the ratio of $r / a$ determines the fragile topological properties of the system with the topological transition critical value of $r / a \approx 0.225$ [39]. Large $r / a$ induces trivial acoustic crystals for which the frequencies of $p$ orbitals are smaller than that of the $d$ orbitals, see Fig. 1(a), where $r / a \approx 0.260$. On the contrary, small $r / a$ leads to topological acoustic crystals for which the frequencies of $p$ orbitals are larger than that of the $d$ orbitals, see Fig. $1(\mathrm{~b})$, where $r / a \approx 0.173$. To confirm the topology of the three bands below the second band gap in Figs. 1(a) and 1(b), the evolutions of their Berry phases are calculated by the Wilson-loop approach [79,80]. Since band 1 is isolated from mutually connected bands 2 and 3, the Berry phase for band 1 can be calculated separately, while those for the rest bands 2 and 3 should be considered together. This calculation way gives the Berry phases in Figs. 1(c) and 1(d), corresponding to Figs. 1(a) and 1(b), respectively. The Berry phases for band 1 in Figs. 1(c) and 1(d) are both pinned at $-\pi$, so that band 1 is trivial. However, the Berry phases for bands 2 and 3 are gaped and gapless, respectively, indicating that bands 2 and 3 are topological in Fig. 1(b). To address the fragility of the topology of bands 2 and 3 in Fig. 1(b), we recalculate the berry phases by considering bands 1, 2, and 3 together [73], see Fig. 1(e). Since the Berry phases is gaped in Fig. 1(e), the topology of bands 2 and 3 is fragile, which has also been confirmed in the recent work [79]. The fragile topology leads to the two branches of the ATESs in the bulk band gap, see the red and blue solid lines in Fig. 1(f). The mini gap at the cross point of the two-branch edge bands indicates that the backscattering of the ATES propagation is not totally immune but largely suppressed in principle.

One of the most intriguing feature of the stable topological insulators is the transport robustness under impurities, while the transport properties for the fragile topology are still unclear up to now. To address this issue, we investigate the transport of the ATESs in the fragile ATI, coupled to one designed acoustic cavity (AC) with the eigenfrequency in the topological band gap. As an example, the single-mode ACs in Fig. 2 are formed by removing five steel rods, which makes sure that the AC eigenfrequencies fall into the topological band gap. When the incident ATES is resonant with the AC mode, the transmissivity always decreases to zero, resulting in a perfect reflection [see the Figs. 2(a)-2(c)]. The zero transmission definitely tells that the ATES transport in the fragile ATI is also fragile. The phase change of the transmitted ATESs due to the AC is shown in the Figs. 2(d)-2(f), whose profiles are Fano form. Thus this fragile transport is fundamentally useful in the control of acoustic wave in a acoustic integrated circuits, since both the amplitude and phase of the transmitted wave can be fully controlled in such a cavitywaveguide coupled system. The fragile transport requires that the cavity eigenfrequency should be in the topological band gap. In photonic systems, one previous work proposed a similar idea to tune the transport of the phonic TESs, but it neglected the relation between the perfect reflection with the system topology [81]. In general, the ATES reflection can be explained as the breaking of the $C_{6 v}$ symmetry near the AC, which breaks the pseudo time-reversal symmetry (TRS) and leads to the pseudospin mixing. However, this explanation does not uncover the origin why the transmission should decrease to zero at the AC eigenfrequency.

\section{TRANSPORT THEORY}

To reveal the origin of the perfect reflection and tunable characteristic, we build a one-dimensional acoustic topological waveguide (ATW)-cavity model to describe the ATES transport in the present section. The Hamiltonian for the coupled architecture of one-dimensional ATW with the AC 


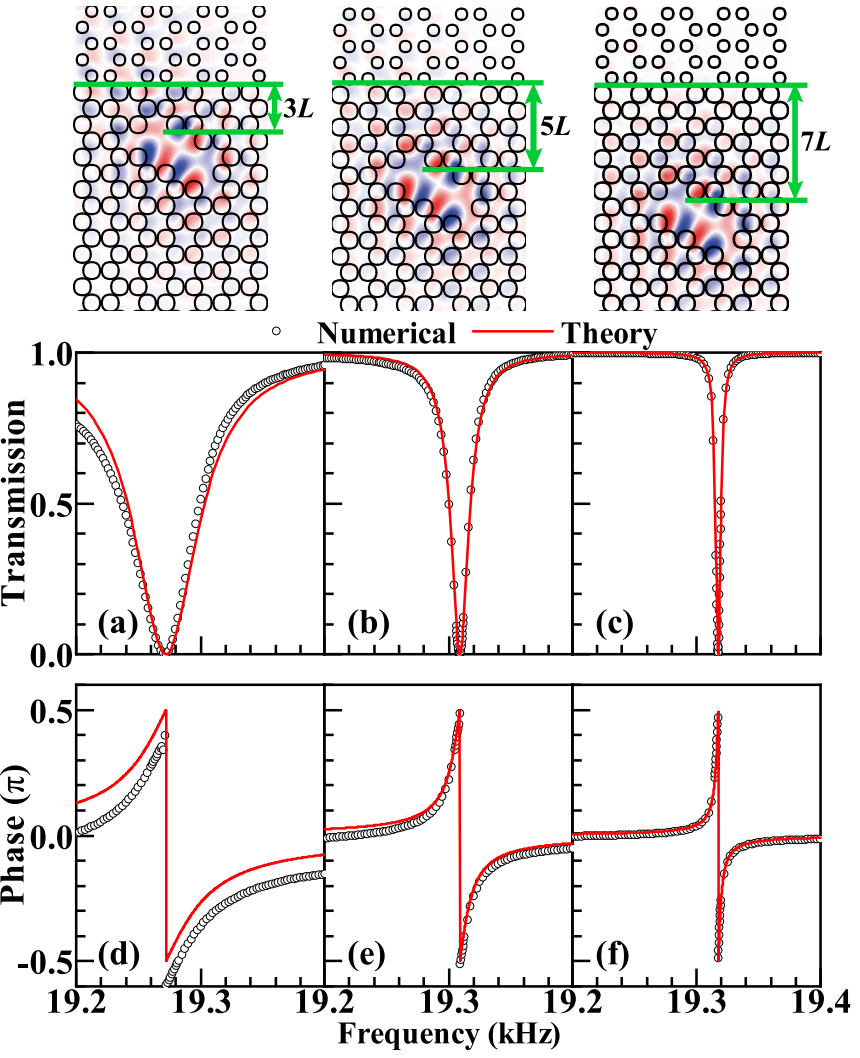

FIG. 2. Top three panels display the topological waveguidecavity coupled architectures and related field distributions that correspond to the zero-transmission frequencies in (a)-(c). [(a)-(c)] Transmission spectra of the topological edge state coupled with one side single-mode acoustic cavity with different distance, corresponding to $3 L, 5 L$, and $7 L$, respectively. [(d)-(f)] Phase change of the transmitted ATESs due to the acoustic cavity, corresponding to (a)-(c), respectively. The acoustic crystals above and below the interface are topological and trivial, respectively. The acoustic cavities are placed in the trivial crystal. Notion of $3 L$ (similar for others) represents the distance between the cavity and waveguide, i.e., three layers of the steel rods. Numerically calculated data of circle dots are fitted by the red curves.

can be written as [82]

$$
H=\iint \hat{\psi}^{\dagger}(x, y) \hat{\omega}(-i \nabla) \hat{\psi}(x, y) d x d y,
$$

where $\hat{\omega}(-i \nabla)$ and $\hat{\psi}(x, y)$ are the operators of the phonon energy and field, respectively. For the structures in the right panels of the Fig. 2, one can separate the phonon field into three parts, namely, the right-moving waveguide field $\hat{\psi}_{R}(x, y)$ with pseudospin up, the left-moving waveguide field $\hat{\psi}_{L}(x, y)$ with pseudospin down, and the cavity field $\hat{\psi}_{c}(x, y)$. The acoustic cavity modes are confined in the $x$ and $y$ directions, while the topological edge states are only limited in the $y$ direction which is perpendicular to the direction of propagation ( $x$ direction). Thus the three parts of the phonon field operator can be expressed as

$$
\begin{aligned}
& \hat{\psi}_{R}(x, y)=\hat{R}(x) \phi_{R}(y)|\uparrow\rangle, \\
& \hat{\psi}_{L}(x, y)=\hat{L}(x) \phi_{L}(y)|\downarrow\rangle, \\
& \hat{\psi}_{c}(x, y)=\hat{c} \phi_{c}(x, y) .
\end{aligned}
$$

where $\hat{R}^{\dagger}(x)$ and $\hat{L}^{\dagger}(x)[\hat{R}(x)$ and $\hat{L}(x)]$ are the creation [annihilation] operators of the rightward- and leftward-moving waveguide modes, corresponding to the pseudospin up and down ATESs, respectively. $\hat{c}^{\dagger}$ is the creation operator of the conventional cavity mode of $\phi_{c}(x, y)$. Since the rightwardand leftward-moving ATESs satisfy the pseudo-TRS, it is natural to take $\phi_{R}(y)=\phi_{L}(y)=\phi_{w}(y)$, describing the spacial limitation along $y$ direction. To omit the first derivative with respect to $y$ in the energy operator of $\hat{\omega}(-i \nabla)$, we make the transform

$$
\begin{aligned}
\hat{\omega}(-i \nabla)\left[\hat{R}(x) \phi_{w}(y)\right] & =\left[\hat{\omega}\left(i \frac{\partial}{\partial x}\right) \hat{R}(x)\right] \phi_{w}(y), \\
\hat{\omega}(-i \nabla)\left[\hat{L}(x) \phi_{w}(y)\right] & =\left[\hat{\omega}\left(-i \frac{\partial}{\partial x}\right) \hat{L}(x)\right] \phi_{w}(y), \\
\hat{\omega}(-i \nabla) \phi_{c}(x, y) & =\omega_{c} \phi_{c}(x, y) .
\end{aligned}
$$

Near the cavity eigenfrequency of $\omega_{c}$ the dispersions of the rightward- and leftward-moving ATESs are linear with respect to the wave vector $k$, i.e., $\omega_{ \pm}=\omega_{c}-v_{g} k_{c} \pm v_{g} k$, where $v_{g}$ is the group velocity and $k_{c}$ is determined by $\left.\omega_{+}\right|_{k=k_{c}}=\omega_{c}$. Substituting Eqs. (2) and (3) into Eq. (1) leads to

$$
H=H_{W}+H_{C}+H_{I},
$$

where $H_{W}, H_{C}$, and $H_{I}$ are the Hamiltonians of the waveguide, cavity and their coupling, respectively. They have the forms

$$
\begin{aligned}
H_{W}= & \int d x \hat{R}^{\dagger}(x)\left(\omega_{c}-v_{g} k_{c}+i v_{g} \frac{\partial}{\partial x}\right) \hat{R}(x) \\
& +\int d x \hat{L}^{\dagger}(x)\left(\omega_{c}-v_{g} k_{c}-i v_{g} \frac{\partial}{\partial x}\right) \hat{L}(x), \\
H_{C}= & \omega_{c} \hat{c}^{\dagger} \hat{c}, \\
H_{I}= & \int d x\left[V_{R}^{*}(x) c^{\dagger} \hat{R}(x)+V_{R}(x) \hat{R}^{\dagger}(x) \hat{c}\right] \\
& +\int d x\left[V_{L}^{*}(x) c^{\dagger} \hat{L}(x)+V_{L}(x) \hat{L}^{\dagger}(x) \hat{c}\right] .
\end{aligned}
$$

The coupling functions of $V_{R}(x)$ and $V_{L}(x)$ are

$$
V_{R}(x)=V_{L}(x)=\omega_{c} \int \phi_{w}^{*}(y) \phi_{c}(x, y) d y .
$$

The relation of $V_{R}(x)=V_{L}(x)$ originates from the pseudoTRS between the rightward- and leftward-moving waveguide modes.

The system eigenstate can be written as

$$
|\Phi\rangle=\int d x\left[\mathcal{R}(x) \hat{R}^{\dagger}(x)+\mathcal{L}(x) \hat{L}^{\dagger}(x)\right]|\varnothing\rangle+\mathcal{C} c^{\dagger}|\varnothing\rangle,
$$

where $|\varnothing\rangle$ represents the vacuum state, with zero excitation either in the cavity or in the waveguide. $C$ is the excitation amplitude of the acoustic cavity mode. $\mathcal{R}(x)$ and $\mathcal{L}(x)$ are the wave functions of the pseudo-spin up and down states, respectively. Since the distribution of the considered cavity mode along the ATW is smaller than the wavelength of the incident acoustic wave, a $\delta$-type coupling between cavity and ATW can be adopted, namely, $V_{R}(x)=\delta(x) V_{R}$ and $V_{L}(x)=$ $\delta(x) V_{L}$. 
Substituting Eqs. (4) and (7) into the Schrödinger equation,

$$
i \hbar \frac{\partial}{\partial t}|\Phi\rangle=H|\Phi\rangle
$$

we can get the coupled equations for $\mathcal{R}(x), \mathcal{L}(x)$ and $\mathcal{C}$ as follows:

$$
\begin{aligned}
i \hbar \frac{\partial}{\partial t} \mathcal{R}(x) & =\left(\omega_{c}-v_{g} k_{c}+i v_{g} \frac{\partial}{\partial x}\right) \mathcal{R}(x)+V_{R} \delta(x) \mathcal{C}, \\
i \hbar \frac{\partial}{\partial t} \mathcal{L}(x) & =\left(\omega_{c}-v_{g} k_{c}-i v_{g} \frac{\partial}{\partial x}\right) \mathcal{L}(x)+V_{L} \delta(x) \mathcal{C}, \\
i \hbar \frac{\partial}{\partial t} \mathcal{C} & =\omega_{c} \mathcal{C}+V_{R} \mathcal{R}(0)+V_{L} \mathcal{L}(0) .
\end{aligned}
$$

For a steady state that the incident acoustic wave has frequency of $\omega$, one can find the transmission coefficient as

$$
t=\frac{2\left(\omega-\omega_{c}\right)+i v_{g}^{-1}\left(\left|V_{L}\right|^{2}-\left|V_{R}\right|^{2}\right)}{2\left(\omega-\omega_{c}\right)+i v_{g}^{-1}\left(\left|V_{L}\right|^{2}+\left|V_{R}\right|^{2}\right)},
$$

by setting the pseudospin up and down states to be

$$
R(x)=e^{-i k x} \theta(-x)+t e^{-i k x} \theta(x), \quad L(x)=r e^{i k x} \theta(-x),
$$

respectively.

According to Eq. (10), one can immediately find $\left.t\right|_{\omega=\omega_{c}}=$ 0 if $\left|V_{L}\right|=\left|V_{R}\right|$, i.e., the perfect reflection regardless of the value of coupling strength between the topological waveguide and the cavity. In fact, the condition of $\left|V_{R}\right|=\left|V_{L}\right|$ is protected by the fragile topology in the present system, dating from the pseudo TRS between the rightward- and leftward-moving waveguide modes, see Eq. (6). Since the condition for the TPPR is just that the ACs can flip the spin of the ATESs, we remark that the TPPR is a hallmark feature of topological systems once the spin-flip mechanism is introduced for them, for examples, spin or valley Hall effect models from optical to elastic waves. In contrast, the TESs exhibit topologically protected perfect transmission in systems with strong topology [35,47,83-87].

Equation (10) shows high effectiveness for describing the transmission amplitude and phase change in Fig. 2, indicating that it is reasonable to take the coupling between the $\mathrm{AC}$ and ATW as $\delta$ type. From the left to right columns in Fig. 2, the fitted values of $\left(\omega_{c}, J\right)$ with $J \equiv\left|V_{R}\right|^{2} / v_{g}=\left|V_{L}\right|^{2} / v_{g}$ are equal to $(19.272 \mathrm{kHz}, 30.930 \mathrm{~Hz}),(19.308 \mathrm{kHz}, 8.777 \mathrm{~Hz})$, and $(19.318 \mathrm{kHz}, 2.357 \mathrm{~Hz})$, respectively. $\omega_{c}$ and $J$ present a little blueshift and a decrease, respectively, since the ATW influence on the $\mathrm{AC}$ decreases with increasing the distance between the AC and ATW. The deviation between the numerical and theoretical results in Figs. 2(a) and 2(d) tell that the non- $\delta$ coupling effects appear between the AC mode and ATESs [82]. Since the AC orientation and size only change the AC eigenfrequency and coupling strength with the ATES, they cannot kill the TPPR, as long as the AC eigenfrequency falls into the topological band gap, but can influence the line width and position of the zero-transmission frequency of the spectra.

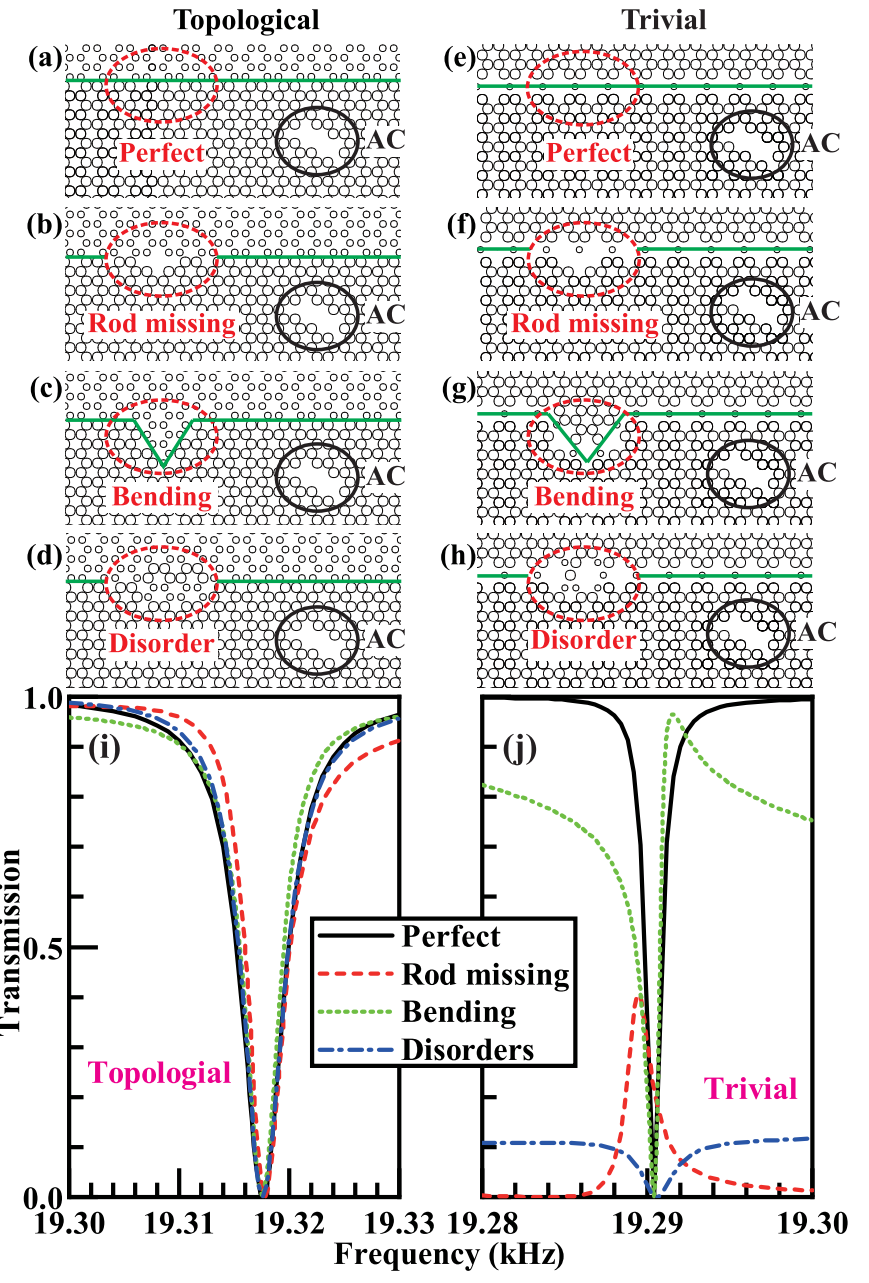

FIG. 3. Schematic diagrams of waveguide-cavity coupling systems with [(a)-(d)] topological and [(e)-(h)] trivial waveguides, where the dashed red and solid black circles denote the added disorders and ACs, respectively. (i) and (j) show the transmission spectra of the topological and trivial waveguide-cavity coupling systems in (a)-(d) and (e)-(h), respectively.

The transmissivity and phase of the ATESs could be adjusted simultaneously, which presents a broad of applications.

\section{APPLICATION OF THE TPPR}

We first confirm the robustness of the TPPR by adding the common fabrication defects on the perfect waveguide (see the Fig. 3), for example, rod missing, bending interface, and disorders. Two types of the waveguide are considered here for comparison, i.e., one is topological and the other is trivial, see their structures in Figs. 3(a)-3(h). For the topological ones the transmission spectra, plotted in Fig. 3(i), are all similar with each other. However, for the trivial ones, the transmission spectra can be strongly modified by adding the afore-mentioned defects, see the line shapes in Fig. 3(j). The results in Fig. 3 thus demonstrate convincingly the TPPR as a unique feature of the TESs due to the fragile topology and its robustness against defects.

Since the above discussed defects and the ACs in Figs. 2 and 3 are all defects in essential, one may wonder what kind 


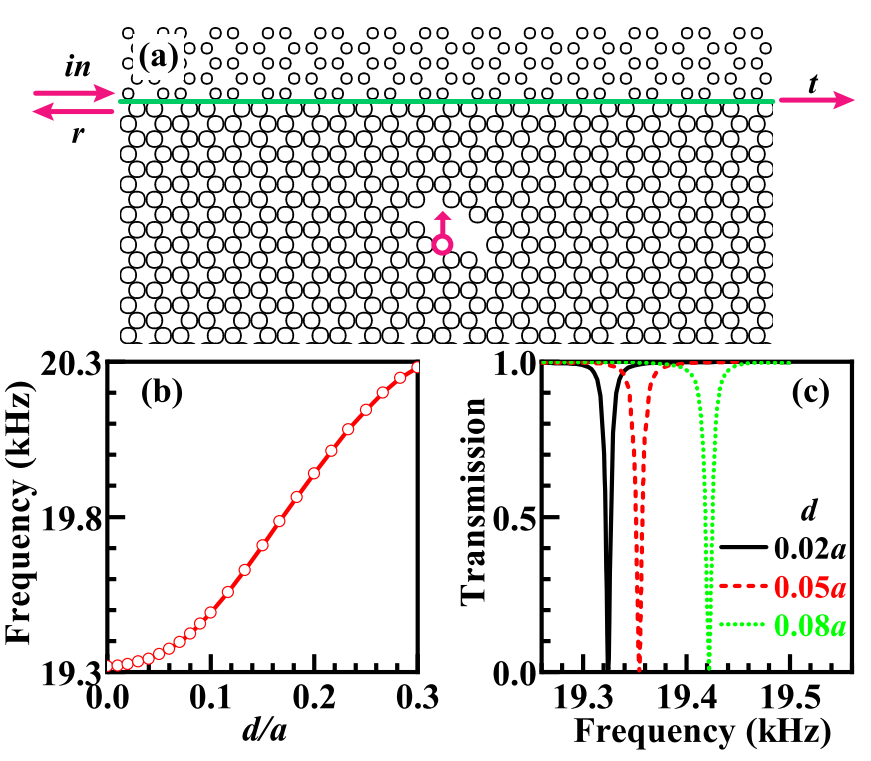

FIG. 4. (a) Schematic draft of topological acoustic devices where the incident ATES with pseudospin up is transmitted and reflected as those with pseudospin up and down, respectively. (b) AC eigenfrequency vs red rod movement distance, $d$, along the vertical direction. (c) Transmission spectra for $d=0.02 a, 0.05 a$, and $0.08 a$.

of defects can or cannot reflect the transport of the ATESs, where the frequencies of the defect modes play a key role. As the frequencies locate in the topological band gap, the defects can reflect the ATES transport, while as the frequencies are outside of the topological band gap, the ATES transport is immune to them, i.e., no pseudospin flip for the ATESs. For the trivial waveguide system, the defects in general can always change the transmission spectra no matter their mode frequencies are in or outside of the trivial band gap, due to the lack of the topological protection. Therefore, one can find the TPPR in the fragile topological insulators. The TPPR of the system is guaranteed by the robust transport of topological edge states, but the ACs here are the common defect-induced cavities whose eigenfrequencies are unstable to the perturbation. That is, if the defect is placed just near the AC, the AC eigenfrequency would be changed and therefore, the TPPR will move to the new $\mathrm{AC}$ eigenfrequency as long as it still falls into the topological band gap. This can be overcome by replacing the present $\mathrm{AC}$ with the topological cavity with eigenfrequency robust against disorders, for example, corner states in high-order topological insulators [65-67,88-93].

Topological acoustics as a novel research field brings about remarkable changes to the applied acoustics based on the topologically protected transmission of the ATESs [[35-37,39,42,49,50],[59,68]]. Here, we demonstrate two potential applications that base on the TPPR, whose schematic structure is shown in Fig. 4(a) where the red steel rod can move along the vertical direction. The rod movement could be achieved by connecting it with an external mechanical arm. The AC eigenfrequency increases with increasing the rod movement distance, $d$, as $d \in(0,0.3) a$, see Fig. 4(b). Accordingly, the transmission spectra of the ATESs presents a blueshift when $d$ increases from $0.02 a$ to $0.08 a$, see Fig. 4(c).

One natural application of this mechanism is the distance sensor, since $d$ determines the position of the transmission dip.
The minimum measurable distance is determined by the half width at half dip and the tangent of the AC frequency shift with respect to $d$. As an example, the minimum measurable distance is $\sim 0.003 a$ at $d=0.05 a$ and is $\sim 0.001 a$ at $d=$ $0.17 a$. On the other hand, the maximum measurable distance is determined by the topological band gap and thereby reads about several tenth of $a$. Since the minimum measurable distance is much less than the lattice constant, this topological sensor has potential for achieving a very high precision measurement.

Another practical application is the topological acoustic switch whose working mechanism can be expressed as follow. For the switch the red rod takes only two positions, for example, $d=0.02 a$ and $d=0.08 a$. When the working frequency is $19.32 \mathrm{kHz}$ [i.e., the position of the transmission dip for $d=$ $0.02 a$, see the solid black curve in Fig. 4(c)], the switch turns on when $d=0.08 a$, while turns off when $d=0.02 a$, with an infinite on/off ratio in theory because of the TPPR at the off state. In practice, the inevitable deviation of $d$ from its value in the off state can decrease the on/off ratio, since the deviation of $d$ can shift the transmission spectra. This blemish could be depressed by increasing the coupling strength between the AC and ATW which makes the transmission dip wider, referred to Fig. 2(a).

In order to show the dynamic response characteristics of the acoustic switch, we use the dynamic Eq. (9) to study the properties of the switch, since it can well describe the transmission spectra of the ATESs coupled with the acoustic cavities. The incident wave is reflected when the switch is in the off state [for which $d=0.02 a$, see Figs. 5(a) and 5(e)], while the incident wave is transmitted [for which $d=0.08 a$, see Figs. 5(b) and 5(f)]. The transition of the switch from off to on is shown in Figs. 5(c) and 5(g) and that from on to off is in Figs. 5(d) and 5(h). From Figs. 5(g) and 5(h), one can read the rise distance, $d_{\uparrow}$, and decay distance, $d_{\downarrow}$, for the probability density in the real space, which give the rise time of $t_{\uparrow}=$ $d_{\uparrow} / v_{g} \approx 3.1 \mathrm{~ms}$ and decay time of $t_{\downarrow}=d_{\downarrow} / v_{g} \approx 4.1 \mathrm{~ms}$. The rise time and decay times are about 60 to 80 times of the working period of the acoustic wave (being about $0.0518 \mathrm{~ms}$ at the frequency of $19.32 \mathrm{kHz}$ ). The switch on/off ratio, defined as the ratio of the transmission probability of the on state to that of the off state, i.e., $\chi=T_{\text {on }} / T_{\text {off }}$, is about 1300 from Figs. 5(e) and 5(f)].

\section{CONCLUSION}

We firstly demonstrated a fragile acoustic topological insulator and then investigated the tuning of the side singlemode cavity on the transport of the topological edge states. The transport of the topological edge states was found to be immune to the defects when their resonant frequencies are outside of the topological band gap, otherwise, the defect can perfectly reflect the topological edge states at the resonant frequency. According to this mechanism, the topologically protected perfect reflection can be achieved by coupling the topological edge states with one single-mode cavity (whose frequency is in the topological band gap), which is immune to the conventional defects, such as bending and disorders. The single-mode cavity can tune the amplitude and phase of the transmitted topological edge states. This 

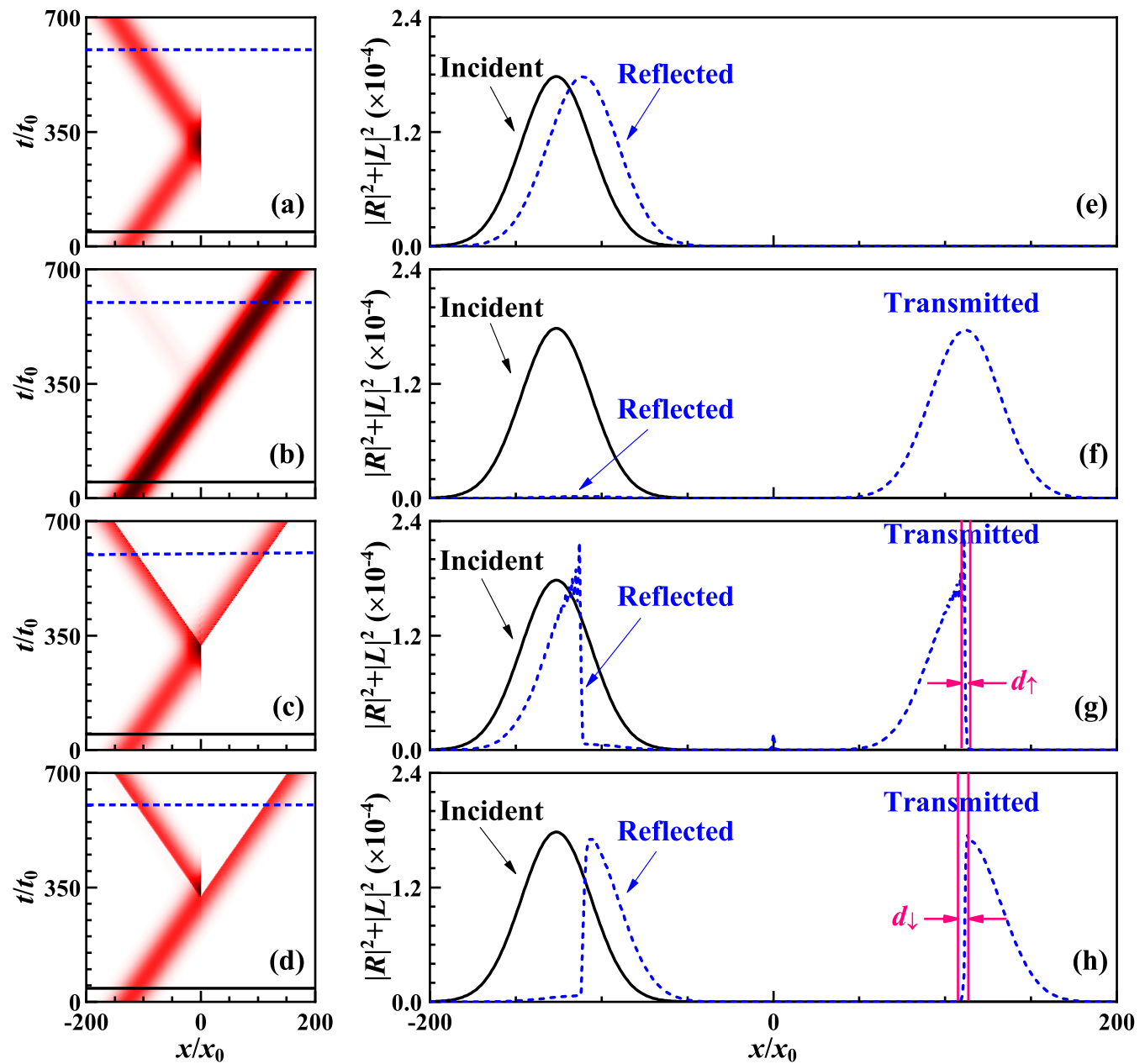

FIG. 5. (Left column) Contour plots of the evolution of the phonon probability density for (a) switch off state, (b) switch on state, (c) switch off to on state, and (d) switch on to off state. (Right column) Distribution of the photon probability at $t=40 t_{0}$ and $600 t_{0}$, corresponding to the black and dashed blue lines in the left panels. The rise and decay distances, i.e., $d_{\uparrow}$ and $d_{\downarrow}$ in $(\mathrm{g})$ and (h), give the rise time $t_{\uparrow}=d_{\uparrow} / v_{g} \approx 3.1 \mathrm{~ms}$ and the decay time $t_{\downarrow}=d_{\downarrow} / v_{g} \approx 4.1 \mathrm{~ms}$, which are about 60 to 80 times of the working period of the acoustic wave (being about $0.0518 \mathrm{~ms}$ at the frequency of $19.32 \mathrm{kHz}$ ). The switch on/off ratio, defined as the ratio of the transmission probability of the on state to that of the off state, i.e., $\chi=T_{\text {on }} / T_{\text {off }}$, reads about 1300 from (a) and (b).

topological transport is well described by the one-dimensional topological waveguide-cavity transport theory, revealing that the perfect reflection is protected by the fragile topology. The fragile topology, combining topological properties and convenient tunability, paves the way for broad applications, such as topological acoustic switches, sensors, and phase modulators.

\section{ACKNOWLEDGMENTS}

This work was supported by the National Natural Science Foundation of China (Grant Nos. 11304015, 11675116, 11734003), National Key R\&D Program of China (Grant No. 2016YFA0300600), and the Jiangsu Province SpeciallyAppointed Professor Funding.
[1] M. S. Kushwaha, P. Halevi, L. Dobrzynski, and B. DjafariRouhani, Phys. Rev. Lett. 71, 2022 (1993).

[2] R. Martínez-Sala, J. Sancho, J. V. Sánchez, V. Gómez, J. Llinares, and F. Meseguer, Nature (London) 378, 241 (1995).

[3] J. V. Sánchez-Pérez, D. Caballero, R. Mártinez-Sala, C. Rubio, J. Sánchez-Dehesa, F. Meseguer, J. Llinares, and F. Gálvez, Phys. Rev. Lett. 80, 5325 (1998).

[4] Z. Liu, X. Zhang, Y. Mao, Y. Zhu, Z. Yang, C. T. Chan, and P. Sheng, Science 289, 1734 (2000).
[5] N. Fang, D. Xi, J. Xu, M. Ambati, W. Srituravanich, C. Sun, and X. Zhang, Nat. Mater. 5, 452 (2006).

[6] M.-H. Lu, C. Zhang, L. Feng, J. Zhao, Y.-F. Chen, Y.-W. Mao, J. Zi, Y.-Y. Zhu, S.-N. Zhu, and N.-B. Ming, Nat. Mater. 6, 744 (2007).

[7] Y. Pennec, J. O. Vasseur, B. Djafari-Rouhani, L. Dobrzyński, and P. A. Deymier, Surf. Sci. Rep. 65, 229 (2010).

[8] S. Zhang, C. Xia, and N. Fang, Phys. Rev. Lett. 106, 024301 (2011). 
[9] J. Mei, G. Ma, M. Yang, Z. Yang, W. Wen, and P. Sheng, Nat. Commun. 3, 756 (2012).

[10] Y. Li, B. Liang, Z.-m. Gu, X.-y. Zou, and J.-c. Cheng, Sci. Rep. 3, 2546 (2013).

[11] P. A. Deymier, Acoustic Metamaterials and Phononic Crystals (Springer Science \& Business Media, 2013), Vol. 173.

[12] G. Ma, M. Yang, S. Xiao, Z. Yang, and P. Sheng, Nat. Mater. 13, 873 (2014).

[13] L. Zigoneanu, B.-I. Popa, and S. A. Cummer, Nat. Mater. 13, 352 (2014).

[14] C. Shen, J. Xu, N. X. Fang, and Y. Jing, Phys. Rev. X 4, 041033 (2014).

[15] M. Yang, C. Meng, C. Fu, Y. Li, Z. Yang, and P. Sheng, Appl. Phys. Lett. 107, 104104 (2015).

[16] A. Khelif and A. Adibi, Phononic Crystals (Springer, 2015).

[17] N. Kaina, F. Lemoult, M. Fink, and G. Lerosey, Nature (London) 525, 77 (2015).

[18] G. Ma and P. Sheng, Sci. Adv. 2, e1501595 (2016).

[19] S.-Y. Yu, X.-C. Sun, X. Ni, Q. Wang, X.-J. Yan, C. He, X.-P. Liu, L. Feng, M.-H. Lu, and Y.-F. Chen, Nat. Mater. 15, 1243 (2016).

[20] J. Christensen, M. Willatzen, V. R. Velasco, and M.-H. Lu, Phys. Rev. Lett. 116, 207601 (2016).

[21] C. Shi, M. Dubois, Y. Wang, and X. Zhang, Proc. Natl. Acad. Sci. USA 114, 7250 (2017).

[22] C. Qiu, Z. Liu, J. Shi, and C. Chan, Appl. Phys. Lett. 86, 224105 (2005).

[23] L.-Y. Wu, L.-W. Chen, and C.-M. Liu, Appl. Phys. Lett. 95, 013506 (2009).

[24] A. H. Safavi-Naeini, J. T. Hill, S. Meenehan, J. Chan, S. Gröblacher, and O. Painter, Phys. Rev. Lett. 112, 153603 (2014).

[25] A. Khelif, A. Choujaa, S. Benchabane, B. Djafari-Rouhani, and V. Laude, Appl. Phys. Lett. 84, 4400 (2004).

[26] J.-H. Sun and T.-T. Wu, Phys. Rev. B 74, 174305 (2006).

[27] M. Oudich, M. B. Assouar, and Z. Hou, Appl. Phys. Lett. 97, 193503 (2010).

[28] J. O. Vasseur, P. A. Deymier, B. Djafari-Rouhani, Y. Pennec, and A. C. Hladky-Hennion, Phys. Rev. B 77, 085415 (2008).

[29] H. Zhu and F. Semperlotti, Phys. Rev. Lett. 117, 034302 (2016).

[30] Y. Pennec, B. Djafari-Rouhani, J. O. Vasseur, H. Larabi, A. Khelif, A. Choujaa, S. Benchabane, and V. Laude, Appl. Phys. Lett. 87, 261912 (2005).

[31] X. Li and Z. Liu, Solid State Commun. 133, 397 (2005).

[32] H. Zhu and F. Semperlotti, AIP Adv. 3, 092121 (2013).

[33] B. Rostami-Dogolsara, M. K. Moravvej-Farshi, and F. Nazari, IEEE Trans. Ultrason. Ferroelectr. Freq. Control 63, 1468 (2016).

[34] B. Rostami-Dogolsara, M. K. Moravvej-Farshi, and F. Nazari, Phys. Rev. B 93, 014304 (2016).

[35] Z. Yang, F. Gao, X. Shi, X. Lin, Z. Gao, Y. Chong, and B. Zhang, Phys. Rev. Lett. 114, 114301 (2015).

[36] A. B. Khanikaev, R. Fleury, S. H. Mousavi, and A. Alù, Nat. Commun. 6, 8260 (2015).

[37] X. Ni, C. He, X.-C. Sun, X.-p. Liu, M.-H. Lu, L. Feng, and Y.-F. Chen, New J. Phys. 17, 053016 (2015).

[38] M. Xiao, W.-J. Chen, W.-Y. He, and C. T. Chan, Nat. Phys. 11, 920 (2015).

[39] C. He, X. Ni, H. Ge, X.-C. Sun, Y.-B. Chen, M.-H. Lu, X.-P. Liu, and Y.-F. Chen, Nat. Phys. 12, 1124 (2016).
[40] Z.-G. Chen and Y. Wu, Phys. Rev. Appl. 5, 054021 (2016).

[41] C. He, Z. Li, X. Ni, X.-C. Sun, S.-Y. Yu, M.-H. Lu, X.-P. Liu, and Y.-F. Chen, Appl. Phys Lett. 108, 031904 (2016).

[42] J. Mei, Z. Chen, and Y. Wu, Sci. Rep. 6, 32752 (2016).

[43] J. Lu, C. Qiu, M. Ke, and Z. Liu, Phys. Rev. Lett. 116, 093901 (2016).

[44] Z. Yang and B. Zhang, Phys. Rev. Lett. 117, 224301 (2016).

[45] Y. Deng, H. Ge, Y. Tian, M. Lu, and Y. Jing, Phys. Rev. B 96, 184305 (2017).

[46] S. Yves, R. Fleury, F. Lemoult, M. Fink, and G. Lerosey, New J. Phys. 19, 075003 (2017).

[47] A. Souslov, B. C. van Zuiden, D. Bartolo, and V. Vitelli, Nat. Phys. 13, 1091 (2017).

[48] S. Shankar, M. J. Bowick, and M. C. Marchetti, Phys. Rev. X 7, 031039 (2017).

[49] L. Ye, C. Qiu, J. Lu, X. Wen, Y. Shen, M. Ke, F. Zhang, and Z. Liu, Phys. Rev. B 95, 174106 (2017).

[50] J. Lu, C. Qiu, L. Ye, X. Fan, M. Ke, F. Zhang, and Z. Liu, Nat. Phys. 13, 369 (2017).

[51] Z. Zhang, Q. Wei, Y. Cheng, T. Zhang, D. Wu, and X. Liu, Phys. Rev. Lett. 118, 084303 (2017).

[52] Z.-G. Chen, J. Zhao, J. Mei, and Y. Wu, Sci. Rep. 7, 15005 (2017).

[53] J. Lu, C. Qiu, W. Deng, X. Huang, F. Li, F. Zhang, S. Chen, and Z. Liu, Phys. Rev. Lett. 120, 116802 (2018).

[54] Y. Shen, C. Qiu, X. Cai, L. Ye, J. Lu, M. Ke, and Z. Liu, Appl. Phys. Lett. 114, 023501 (2019).

[55] M. Wang, L. Ye, J. Christensen, and Z. Liu, Phys. Rev. Lett. 120, 246601 (2018).

[56] Y. Zhu, Y. Peng, X. Fan, J. Yang, B. Liang, X. Zhu, and J. Cheng, arXiv:1801.07942.

[57] Y. Yang, H. Jiang, and Z. H. Hang, Sci. Rep. 8, 1588 (2018).

[58] B.-Z. Xia, S.-J. Zheng, T.-T. Liu, J.-R. Jiao, N. Chen, H.-Q. Dai, D.-J. Yu, and J. Liu, Phys. Rev. B 97, 155124 (2018).

[59] Z. Zhang, Y. Tian, Y. Cheng, Q. Wei, X. Liu, and J. Christensen, Phys. Rev. Appl. 9, 034032 (2018).

[60] Y. Yang, Z. Yang, and B. Zhang, J. Appl. Phys. 123, 091713 (2018).

[61] X. Wen, C. Qiu, J. Lu, H. He, M. Ke, and Z. Liu, J. Appl. Phys. 123, 091703 (2018).

[62] C. He, S.-Y. Yu, H. Ge, H. Wang, Y. Tian, H. Zhang, X.-C. Sun, Y. Chen, J. Zhou, M.-H. Lu et al., Nat. Commun. 9, 4555 (2018).

[63] H. He, C. Qiu, L. Ye, X. Cai, X. Fan, M. Ke, F. Zhang, and Z. Liu, Nature (London) 560, 61 (2018).

[64] F. Li, X. Huang, J. Lu, J. Ma, and Z. Liu, Nat. Phys. 14, 30 (2018).

[65] X. Ni, M. Weiner, A. Alù, and A. B. Khanikaev, Nat. Mater. 18, 113 (2019).

[66] H. Xue, Y. Yang, F. Gao, Y. Chong, and B. Zhang, Nat. Mater. 18, 108 (2019).

[67] X. Zhang, H.-X. Wang, Z.-K. Lin, Y. Tian, B. Xie, M.-H. Lu, Y.-F. Chen, and J.-H. Jiang, Nat. Phys. 15, 582 (2019).

[68] Z. Zhang, Y. Tian, Y. Wang, S. Gao, Y. Cheng, X. Liu, and J. Christensen, Adv. Mater. 30, 1803229 (2018).

[69] Z. Wang, F.-K. Liu, S.-Y. Yu, S.-L. Yan, M.-H. Lu, Y. Jing, and Y.-F. Chen, J. Appl. Phys. 125, 044502 (2019).

[70] B. Bahari, A. Ndao, F. Vallini, A. El Amili, Y. Fainman, and B. Kanté, Science 358, 636 (2017). 
[71] G. Harari, M. A. Bandres, Y. Lumer, M. C. Rechtsman, Y. D. Chong, M. Khajavikhan, D. N. Christodoulides, and M. Segev, Science 359, eaar4003 (2018).

[72] M. A. Bandres, S. Wittek, G. Harari, M. Parto, J. Ren, M. Segev, D. N. Christodoulides, and M. Khajavikhan, Science 359, eaar4005 (2018).

[73] H. C. Po, H. Watanabe, and A. Vishwanath, Phys. Rev. Lett. 121, 126402 (2018).

[74] H. C. Po, L. Zou, T. Senthil, and A. Vishwanath, Phys. Rev. B 99, 195455 (2019).

[75] B. Bradlyn, Z. Wang, J. Cano, and B. A. Bernevig, Phys. Rev. B 99, 045140 (2019).

[76] Z. Song, L. Elcoro, N. Regnault, and B. A. Bernevig, arXiv:1905.03262.

[77] M. B. de Paz, M. G. Vergniory, D. Bercioux, A. García-Etxarri, and B. Bradlyn, Phys. Rev. Res. 1, 032005 (2019).

[78] A. Alexandradinata, J. Höller, C. Wang, H. Cheng, and L. Lu, arXiv: 1908.08541

[79] H.-X. Wang, G.-Y. Guo, and J.-H. Jiang, New J. Phys. 21, 093029 (2019).

[80] R. Yu, X. L. Qi, A. Bernevig, Z. Fang, and X. Dai, Phys. Rev. B 84, 075119 (2011).

[81] C.-Y. Ji, G.-B. Liu, Y. Zhang, B. Zou, and Y. Yao, Phys. Rev. A 99, 043801 (2019).
[82] Y. Zhang and B. Zou, Phys. Rev. A 89, 063815 (2014).

[83] F. D. M. Haldane and S. Raghu, Phys. Rev. Lett. 100, 013904 (2008).

[84] Z. Wang, Y. D. Chong, J. D. Joannopoulos, and M. Soljačić, Phys. Rev. Lett. 100, 013905 (2008).

[85] Z. Wang, Y. Chong, J. D. Joannopoulos, and M. Soljačić, Nature (London) 461, 772 (2009).

[86] P. Wang, L. Lu, and K. Bertoldi, Phys. Rev. Lett. 115, 104302 (2015).

[87] Y. Ding, Y. Peng, Y. Zhu, X. Fan, J. Yang, B. Liang, X. Zhu, X. Wan, and J. Cheng, Phys. Rev. Lett. 122, 014302 (2019).

[88] F.-F. Li, H.-X. Wang, Z. Xiong, Q. Lou, P. Chen, R.-X. Wu, Y. Poo, J.-H. Jiang, and S. John, Nat. Commun. 9, 2462 (2018).

[89] B.-Y. Xie, H.-F. Wang, H.-X. Wang, X.-Y. Zhu, J.-H. Jiang, M.-H. Lu, and Y.-F. Chen, Phys. Rev. B 98, 205147 (2018).

[90] B.-Y. Xie, G.-X. Su, H.-F. Wang, H. Su, X.-P. Shen, P. Zhan, M.-H. Lu, Z.-L. Wang, and Y.-F. Chen, Phys. Rev. Lett. 122, 233903 (2019).

[91] H. Xue, Y. Yang, G. Liu, F. Gao, Y. Chong, and B. Zhang, Phys. Rev. Lett. 122, 244301 (2019).

[92] M. Weiner, X. Ni, M. Li, A. Alù, and A. B. Khanikaev, arXiv:1903.00428.

[93] X.-D. Chen, W.-M. Deng, F.-L. Shi, F.-L. Zhao, M. Chen, and J.-W. Dong, Phys. Rev. Lett. 122, 233902 (2019). 